\title{
Integrating Homeopathy into National Health Mission of India
}

\section{Opinion}

Healthcare delivery in India is passing through a phase of challenging complexity for various reasons. These include paradigm shift in demography, impact of globalisation, changing cultural practices and health beliefs, economic factors and technological innovations. This has not only given rise to multiple options to seek healthcare, but also developed a syncretic and pluralistic model of healthcare. India offers a rich composition of varied healthcare practices, ranging from western biomedical model to the indigenous therapeutic practices and experiences. Each of these models has its strengths and opportunities, which are often complementary to one another.

In the past, the western medical model held sway over the framing of health policies and by default positioned as the official healthcare model. The extensive and often indiscriminate use of this biomedical model, with the chemically modulated drugs has not only exposed the chinks in its limited scope as a dominant system but also bred drug resistance among vast sections of population. The realisation of such catastrophic impact has led to the birth of rational drug use among the practitioners of western medicine. It also has made a collateral impact for increased awareness, interest and acceptance of the indigenous healthcare practices. The perceived safety levels of indigenous practices where the medicines are mostly drawn from natural sources also have been recognised by an increasing majority of patients.

It therefore is both a responsibility and opportunity for health policy makers to evolve a unified model of healthcare that leverages the best practices of all systems. The earlier model of National Rural Health Mission that was unveiled in the year 2005 by the Government of India made a valiant attempt to achieve integration with its motto - 'mainstreaming of AYUSH'. As the idea was new and evidences were not many, it got localised on the fringes of the Mission and very less attention was paid to objectively explore this dimension of healthcare

However, empirical data that is available in the form of case records, hospital attendance, camp registrations, etc, point to potentially competent interventions through the various AYUSH systems.

Homeopathy is classified as part of AYUSH domain. Even though homeopathy originated in Germany in the eighteenth century, this system of medicine found more patronage in India because of its compatibility with eastern philosophical discourse. India has the most number of homeopathy colleges that offer university level education, the highest number of practitioners and the largest global base of population who actively prefer homeopathy. Studies on the efficacy of homeopathy in various clinical conditions with rigorous protocols have established its suitability for a range of disorders spanning across acute and chronic states. Homeopathy

Opinion
Molume 6 Issue 1 - 2017
Mepartmir Ahment of Homeopathy, Rajiv Gandhi University of Health
Sciences, India
*Corresponding author: Munir Ahmed R, Homeopathy,
Director, College Development Council, Rajiv Gandhi
University of Health Sciences, Jayanagar 4th T Block,
Bangalore, Karnataka, 560041, India,
Email: munir.bangalore@gmail.com
Received: February 16, 2017 | Published: March 20, 2017

is also preferred because of its most user-friendly dosage form especially among children. Another edge that homeopathy enjoys is on the economic front; it is perceived as the post economical system of medicine and also the most eco-friendly medicine.

In order to leverage the benefits of experiences from the National Health Campaigns launched in NRHM, we need to focus on the plausible factors that facilitate dissemination of the innovations across the spectrum of program implementation. The goals of National Health Mission are committed to improve the health indicators including those for infant and maternal mortality rates. As part of NRHM, homeopathy was entrusted with the running of National Campaign for Mother and Child Health. This was a pan India event that included policy dialogues, social campaigns, sensitisation of health administrators to the strengths of homeopathy in mother and child care, capacity-building homeopathic professionals in various aspects of mother and child care, sharing of the experiences and successes in the public and professional platforms.

The outcomes of these campaigns are a rich source of information for aligning homeopathy into the national health policy for mother and child health. As observed earlier, the economic edge that homeopathy has along with the documented successes in the domain of mother and child health make a strong case for a niche position for homeopathy in national health policy. To ensure national health services delivery to accelerate gains in health outcomes it must continuously adapt and evolve according to the emerging health landscape. The possibilities offered by outcomes of the quoted national campaign makes a compelling case for change policy review.

It has been observed in a WHO Working Document that sufficient funding and efficacious technology may be necessary 
conditions for achieving health gains, but experience in many countries confirms that they are not sufficient. Effective and efficient service delivery is the point at which the potential of the health system to improve lives meets the opportunity to realise health gains. Health service-delivery performance means access and use by those in need; adequate quality of care to produce health benefits; efficient use of scarce resources; and organizations that can learn, adapt, and improve for thefuture. All too often, potential benefits are not realized because service delivery underperforms. Organizations must combine financial, physical, and human resources to deliver health services.

In the backdrop of this, the following suggestions are offered in the national interest

i. Introduce homeopathy as an option for mother and child health in the national health policy document.

ii. Identify areas of strength and opportunities for homeopathy in mother and child health.

iii. Capacity-build the homeopathic practitioners to effectively contribute their services. iv. Prepare SOPs for uniform and consistent practices by all hierarchy of professionals.

v. Orient the paramedical staff on the use and application of homeopathic medicines.

vi. Familiarise the Anganwadi and ASHA workers to utilise homeopathic medicines in the conditions that are approved for it.

vii. Sensitise the health administrators at the state, district, taluka and block levels to be open and unbiased to adapt homeopathy in the situations that are compatible.

viii. Provide sufficient stocks of homeopathic medicines in the points of distribution.

ix. Constitute an Advisory Committee consisting of homeopathic professionals to assist in the effective implementation of the programs.

$\mathrm{x}$. Institute mechanism for Monitoring and Evaluation, especially on the economic dimension to measure the Return on Investment, so as to extend the project on a larger scale and into newer areas. 